PAPER

\title{
Finite integration technique for coupled acoustic and elastic wave simulation and its application to noncontact ultrasonic testing
}

\author{
Kazuyuki Nakahata, ${ }^{1, *}$, Junjie Chang ${ }^{2}$, Masakazu Takahashi ${ }^{2}$, \\ Katsumi Ohira ${ }^{2}$ and Yukio Ogura ${ }^{2}$ \\ ${ }^{1}$ Department of Civil and Environmental Engineering, Ehime University, \\ 3 Bunkyo, Matsuyama, Ehime, 790-8577 Japan \\ ${ }^{2}$ Japan Probe, Co., Ltd., 14-1-1 Nakamura, Yokohama, 232-0033 Japan
}

(Received 3 October 2013, Accepted for publication 4 April 2014)

\begin{abstract}
To better understand wave propagation and scattering phenomena in noncontact ultrasonic testing (UT), we have developed a time domain simulation tool to model wave propagation in air and solids. The tool is based on the finite integration technique (FIT) and an image-based modeling approach. The image-based FIT can treat the calculation of acoustic, elastic, and coupled waves in a unified grid form. In this paper, we first investigate the accuracy of the interface condition in FIT modeling by comparing numerical results with analytical solutions of transmission and reflection coefficients. Then we validate the FIT using measured signals in guided wave testing with air-coupled transducers. To simulate noncontact UT, a fine mesh is required in the FIT because the wavelength in air is much smaller than that in a solid. To solve the numerical problem with the finer mesh at high speed, we introduce a parallel computation technique with general-purpose computing on graphics processing units (GPUs). It is shown that the noncontact UT simulation can be executed within a reasonable time and with reasonable accuracy using multiple GPUs.
\end{abstract}

Keywords: Simulation, Image-based modeling, Finite integration technique, Acoustic and elastic waves, GPU calculation, Noncontact ultrasonic testing

PACS number: 43.55.Ka, 43.35.Zc, 43.20.El, 43.20.Gp [doi:10.1250/ast.35.260]

\section{INTRODUCTION}

A noncontact ultrasonic testing (UT) system [1] with a high-sensitivity narrowband air-coupled ultrasonic transducer was developed in the early 1990s. The use of aircoupled ultrasonic transducers is feasible and efficient in nondestructive testing (NDT) because it is both noncontact and noncontaminating. In the last decade, this technique was applied in UT for not only metals [2] but also composite materials [3,4]. Recently air-coupled transducers, having a wideband frequency between 0.1 and $2 \mathrm{MHz}$, have been employed for commercial use [5]. Most of the new applications have been made possible by major technological advances in components such as transducers, pulsers, preamplifiers, signal-conditioning, and impedance matching and noise-matching circuits. However, the characteristic acoustic impedance of air is low, resulting in large transmission losses at the interface between air and the solid compared with a fluid or resin couplant.

*e-mail: nakahata@cee.ehime-u.ac.jp
Simulation tools to model ultrasonic wave propagation including the refraction, reflection, and mode conversion at a solid-air interface, and to model the scattering caused by defects can improve the reliability of noncontact UT [6,7]. In simulations, we need the coupling between two linear wave equations: the scalar-valued equation concerning the propagation of acoustic waves and the vector-valued equation modeling the propagation of waves in elastic media. In our previous research, we developed 2D and 3D simulation tools [8] to study elastic wave propagation in the time domain. These tools are based on the finite integration technique $[9,10]$ (FIT) and image-based modeling [11]. The mesh size must be determined according to the smallest wavelength in the target media. Since the wavelength in air is smaller than that in a solid, the noncontact UT simulation requires a finer mesh. To model noncontact UT, numerical methods in the frequency domain need a large computational memory in order to calculate inverse matrices. However, the FIT is effective for UT simulations, especially noncontact UT simulations, which require a huge number of meshes, because the FIT is 
an explicit numerical method using a computational mesh with a constant size.

An ultrasonic wave in air propagates as an acoustic wave, however it propagates as an elastic wave in a solid. In this study, we develop a new image-based FIT code that can simulate linear acoustic, elastic, and coupled waves. In the new FIT, the calculation of acoustic and elastic waves can be treated in a unified grid form. By introducing a unified grid, we can use $2 \mathrm{D}$ vector graphics or $3 \mathrm{D}$ digital images as the input data of the FIT. Indeed, we can feed a digital image into the FIT simulation without any burdensome image processing.

As mentioned above, the noncontact UT simulation requires a finer mesh than contact UT simulations. The use of a finer mesh leads to increased calculation time. To overcome this difficulty, we introduce a parallel calculation technique using general-purpose computing on GPUs. Since the FIT is based on the leap-frog time-marching scheme and a stencil computation [12], fine-grained parallel computing using GPUs can show a good performance. The detail of the accelerated FIT for shear horizontal ( $\mathrm{SH}$ ) wave propagation computed with GPUs was reported in our previous paper [13]. In this paper, we apply the GPU computing technique to the new FIT simulation of elastic and acoustic waves, and show the increased calculation speed. Before running the noncontact UT simulations, we verify the interface condition in the FIT simulation using the analytical theory of the reflection and transmission of plane waves. Next, simulations of noncontact UT are performed using a PC with three GPU boards. Since air-coupled transducers are mostly applied to guided wave testing [2-4], we compare the results of the FIT simulations with experimentally measured signals in guided wave testing.

\section{IMAGE-BASED FINITE INTEGRATION TECHNIQUE}

\subsection{Spatially Discrete Form}

In this section, we outline the FIT approach for the analysis of coupled elastic and acoustic waves. The target areas are assumed to be linear elastic and acoustic fields that satisfy small-deformation theory. The Cartesian coordinates $\left(x_{1}, x_{2}, x_{3}\right)$ are considered. The governing equations of the elastic wave are the constitutive law and the Cauchy equation of motion. These equations are given in integral form for a finite volume $V$ with surface $S$ by

$$
\begin{gathered}
\int_{V} \frac{\partial \tau_{k l}}{\partial t}(\boldsymbol{x}, t) \mathrm{d} V=\int_{S} c_{i j k l}(\boldsymbol{x}) v_{i}(\boldsymbol{x}, t) n_{j}(\boldsymbol{x}) \mathrm{d} S \\
\int_{V} \rho(\boldsymbol{x}) \frac{\partial v_{i}}{\partial t}(\boldsymbol{x}, t) \mathrm{d} V=\int_{S} \tau_{i j}(\boldsymbol{x}, t) n_{j}(\boldsymbol{x}) \mathrm{d} S \\
+\int_{V} f_{i}(\boldsymbol{x}, t) \mathrm{d} V
\end{gathered}
$$

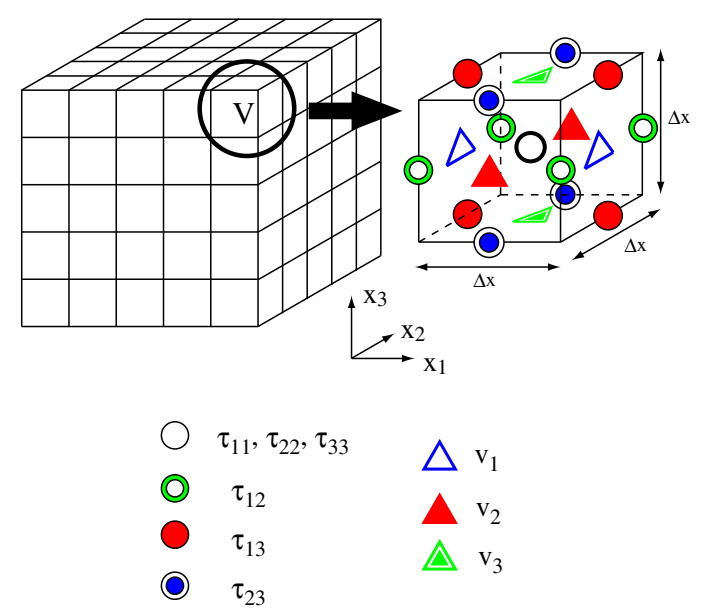

Fig. 1 Finite volume $V$ and allocation of physical quantities in the 3D FIT.

where $\boldsymbol{v}$ is the particle velocity vector, $\boldsymbol{\tau}$ is the stress tensor of the second rank, $\rho$ is the density, $\boldsymbol{n}$ is the outward normal vector on the surface $S$, and $\boldsymbol{f}$ is the body force vector. In Eq. (1), $c$ is the stiffness tensor of rank four. In the case of isotropic materials, $c$ can be written as

$$
c_{i j k l}=\lambda \delta_{i j} \delta_{k l}+\mu\left(\delta_{i k} \delta_{j l}+\delta_{i l} \delta_{j k}\right)
$$

in terms of the two Lamé constants, $\lambda$ and $\mu$. In Eq. (3), $\delta$ is the Kronecker delta tensor, and the summation convention is used. The pressure $(\mathrm{P})$ and shear vertical $(\mathrm{S})$ wave velocities in a solid [s] are given as follows:

$$
c_{\mathrm{P}}^{[\mathrm{s}]}=\sqrt{\frac{\lambda+2 \mu}{\rho}}, \quad c_{\mathrm{S}}^{[\mathrm{s}]}=\sqrt{\frac{\mu}{\rho}} .
$$

We consider a discrete form of Eq. (1) using a cubic voxel as an integral volume $V$ as shown in Fig. 1. Using a voxel with the same size, we can feed a digital image directly into the FIT simulation without any burdensome image processing [11]. Assuming that $\tau_{11}$ is constant in $V$, we have the following:

$$
\begin{aligned}
\dot{\tau}_{11} \Delta x^{3}= & (\lambda+2 \mu)\left[v_{1}^{(\mathrm{R})}-v_{1}^{(\mathrm{L})}\right] \Delta x^{2} \\
& +\mu\left[v_{2}^{(\mathrm{B})}-v_{2}^{(\mathrm{F})}+v_{3}^{(\mathrm{U})}-v_{3}^{(\mathrm{D})}\right] \Delta x^{2},
\end{aligned}
$$

where $\dot{\tau}_{11}=\partial \tau_{11} / \partial t$ and $\Delta x$ is the length of a side in $V$. In Eq. (5), superscripts denote the positions of the physical quantities of the integral volume as shown in Fig. 2. Similarly, the discretization of Eq. (2) gives

$$
\bar{\rho} \dot{v}_{1} \Delta x^{3}=\left[\tau_{11}^{(\mathrm{R})}-\tau_{11}^{(\mathrm{L})}+\tau_{12}^{(\mathrm{B})}-\tau_{12}^{(\mathrm{F})}+\tau_{13}^{(\mathrm{U})}-\tau_{13}^{(\mathrm{D})}\right] \Delta x^{2},
$$

where we let $f_{1}=0$. Since the density $\rho$ is given in the $\tau_{11}$-integration volume as shown in Fig. 2(a), the average value $\bar{\rho}=\left(\rho^{(\mathrm{L})}+\rho^{(\mathrm{R})}\right) / 2$ is used in the integration of $v_{1}$ in Eq. (6). A similar formulation is adopted for the other quantities $\boldsymbol{\tau}$ and $\boldsymbol{v}$. 


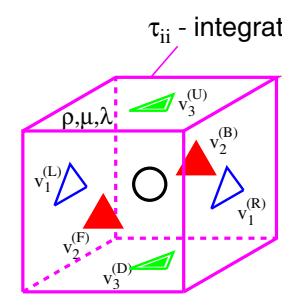

(a)

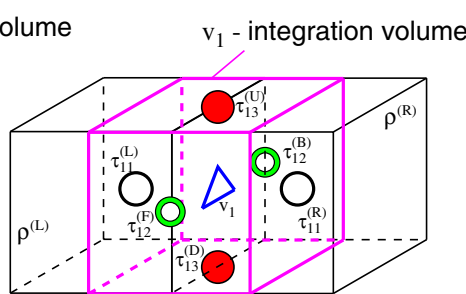

(b)
Fig. $2 \tau_{i i}$-integration volume (a) and $v_{1}$-integration volume (b). All material parameters are defined in the $\tau_{i i}$-integration volume.

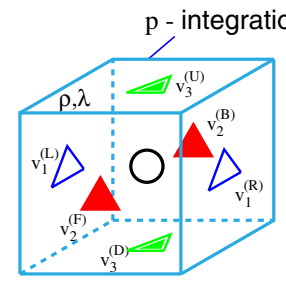

(a)

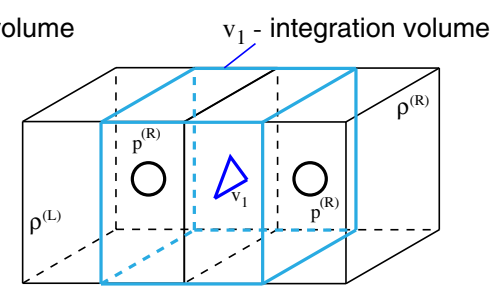

(b)
Fig. $3 p$-integration volume (a) and $v_{1}$-integration volume (b).

In the case of an acoustic field, the governing equations are as follows:

$$
\begin{aligned}
& \int_{V} \frac{\partial p}{\partial t}(\boldsymbol{x}, t) \mathrm{d} V=-\int_{S} K(\boldsymbol{x}) v_{i}(\boldsymbol{x}, t) n_{i}(\boldsymbol{x}) \mathrm{d} S, \\
& \int_{V} \rho(\boldsymbol{x}) \frac{\partial v_{i}}{\partial t}(\boldsymbol{x}, t) \mathrm{d} V=-\int_{S} p(\boldsymbol{x}, t) n_{i}(\boldsymbol{x}) \mathrm{d} S,
\end{aligned}
$$

where $K$ and $p$ are the bulk modulus and pressure, respectively. The P-wave velocity in the acoustic field [a] is given as

$$
c_{\mathrm{P}}^{[\mathrm{a]}]}=\sqrt{\frac{K}{\rho}} .
$$

The discrete form of Eq. (7) and a component of Eq. (8) are expressed with the symbols shown in Fig. 3 as follows:

$$
\begin{aligned}
& \dot{p} \Delta x^{3}=-K\left[v_{1}^{(\mathrm{R})}-v_{1}^{(\mathrm{L})}+v_{2}^{(\mathrm{B})}\right. \\
&\left.-v_{2}^{(\mathrm{F})}+v_{3}^{(\mathrm{U})}-v_{3}^{(\mathrm{D})}\right] \Delta x^{2} \\
& \bar{\rho} \dot{v}_{1} \Delta x^{3}=-\left[p^{(\mathrm{R})}-p^{(\mathrm{L})}\right] \Delta x^{2}
\end{aligned}
$$

Comparing Fig. 2 with Fig. 3, we find an analogy for the grid allocation. More specifically, the positions of the velocity components are the same, and the normal stress $\tau_{i i}$ in the elastodynamic field corresponds to the pressure $p$ in the acoustic field. Therefore, we can use a common grid in the computation by replacing $\tau_{i i}$ with $-p$ (no sum for $i$ ). Since the shear stress in the acoustic field is zero $\left(\tau_{i j}=0(i \neq j)\right)$, the components must vanish as shown in Fig. 3.

\subsection{Time Discretization}

In the time domain, the stress components $\tau$ are allocated at half-time steps, while the velocities $\boldsymbol{v}$ are allocated at full-time steps. The following time discretization yields an explicit leap-frog scheme:

$$
\{\boldsymbol{\tau}\}^{z+\frac{1}{2}}=\{\boldsymbol{\tau}\}^{z-\frac{1}{2}}+\Delta t\{\dot{\boldsymbol{\tau}}\}^{z}, \quad\{\boldsymbol{v}\}^{z}=\{\boldsymbol{v}\}^{z-1}+\Delta t\{\dot{\boldsymbol{v}}\}^{z-\frac{1}{2}},
$$

where $\Delta t$ is the time interval and the superscript $z$ denotes the integer number of the time step. The FIT repeats the operations in Eq. (12) from $z=1$ to $N$ under suitable initial and boundary conditions. A specific stability condition [14] (the Courant-Friedrichs-Lewy (CFL) condition) and adequate spatial resolution [8] are required to calculate the FIT accurately.

\subsection{Interface and Boundary Conditions}

When modeling noncontact UT, it is essential to satisfy the following continuity conditions between the solid [s] and air [a] interface:

$$
v_{N}^{[\mathrm{a}]}=v_{N}^{[\mathrm{s}]}, \quad-p^{[\mathrm{a}]}=\tau_{N N}^{[\mathrm{s}]}, \quad \tau_{N T}^{[\mathrm{s}]}=0,
$$

where subscripts $N$ and $T$ are the normal and tangential directions on the interface, respectively.

Concerning the boundary condition on the surface of the target, a perfectly matched layer (PML), a periodic condition, or the free-surface conditions

$$
\tau_{i j}^{[\mathrm{s}]} n_{j}=0, \quad p^{[\mathrm{a}]}=0
$$

are adopted.

\subsection{Acceleration of the FIT by GPU Computing}

To accelerate the image-based FIT, we use a programming interface, Compute Unified Device Architecture (CUDA [15], Version 5.0), which can control multiple cores in NVIDIA GPUs. CUDA kernels are executed by partitioning the computation into a grid. Typically, a GPU is assigned to a grid, and each grid is divided into blocks composed of atomic calculation units, i.e., threads. We show an example of a computational array of the imagebased FIT and thread assignment in Fig. 4(a). In each block, a thread is associated with a calculation of a component of $\tau_{11}$. Since the block size is limited by the amount of memory available, i.e., $6 \mathrm{~GB}$ on a Tesla $\mathrm{C} 2075$ NVIDIA, we need to use multiple GPUs for large problems. As shown in Fig. 4(b), one CPU ties up one GPU, i.e., one CPU defines a process to manage one GPU. For the communication of data, we use the Message Passing Interface (MPI) library and create outer grid layers for MPI buffers [16]. 


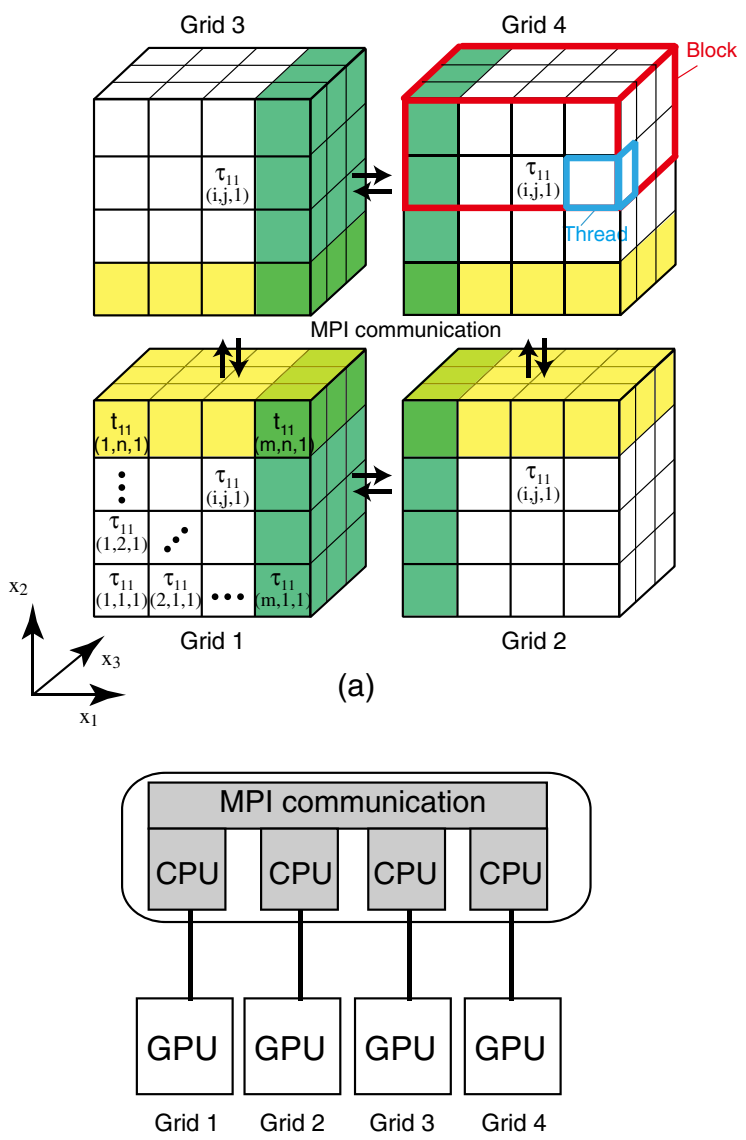

(b)

Fig. 4 (a) Hierarchic structure (grid, block, and thread) in CUDA. (b) The array data at the sides of each grid are communicated using the MPI library.

\section{VERIFICATION OF INTERFACE CONDITION IN FIT SIMULATION}

The performance of the FIT for the elastodynamic scattering problem has already been verified [10]. We have reported the stability in updating solutions using a single precision calculation in the FIT in our previous paper [13]. In this paper we investigate the accuracy of the numerical condition at an air-solid interface in the 3D FIT simulation with the single precision calculation. The reflection and transmission at the plane interface are calculated. The model dimensions used for the accuracy check are $20 \mathrm{~mm} \times 20 \mathrm{~mm} \times 45 \mathrm{~mm}$ (see Fig. 5). The upper part is air (acoustic field; $c_{\mathrm{P}}^{[\mathrm{a}]}=340 \mathrm{~m} / \mathrm{s}$ and $\rho=1.2 \mathrm{~kg} / \mathrm{m}^{3}$ ) and the lower part is aluminum (elastodynamic field; $c_{\mathrm{P}}^{[\mathrm{s}]}=$ $6,360 \mathrm{~m} / \mathrm{s}, c_{\mathrm{S}}^{[\mathrm{s}]}=3,040 \mathrm{~m} / \mathrm{s}$, and $\left.\rho=2,700 \mathrm{~kg} / \mathrm{m}^{3}\right)$. Pressure is induced at the top surface of the model, and a plane $\mathrm{P}$-wave with $0.5 \mathrm{MHz}$ center frequency is transmitted into the air. We choose $\Delta x=0.01 \mathrm{~mm}$ and $\Delta t=1.1 \mathrm{~ns}$ by considering the CFL condition. The continuous conditions in Eq. (14) are implemented at the interface, and periodic boundary conditions are used at the lateral boundaries. The
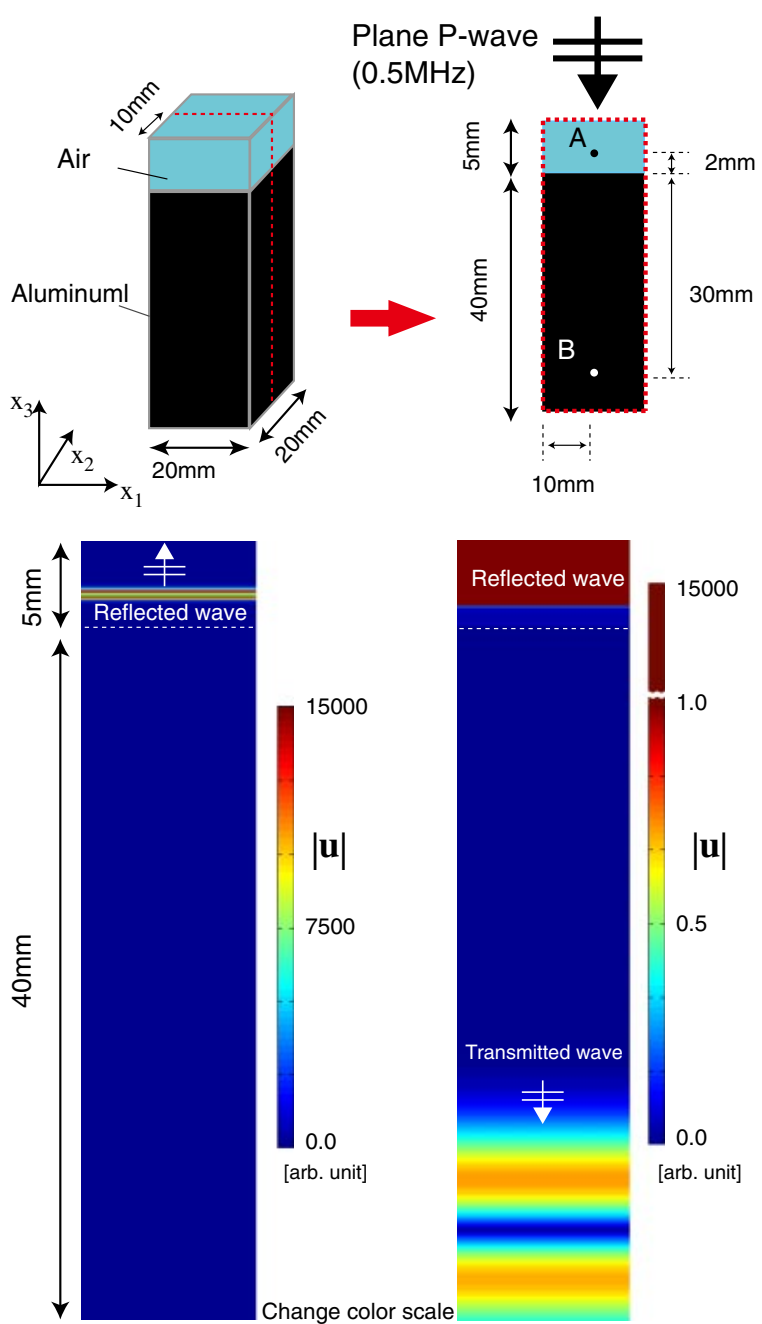

(a)

$(x 1 / 15000)$

(b)

Fig. 5 Numerical model for the verification of the interface condition in the FIT simulation. A plane wave arrives at an air-aluminum interface, and transmitted and reflected waves are generated. The absolute value of the displacement vector $|\boldsymbol{u}|$ is visualized at $t=22.2 \mu \mathrm{s}$. The bottom figures show the wave fields at the same time. When we reduce the value corresponding to the red color, the transmitted wave in aluminum appears. At that time, the reflected wave is saturated with the red color.

bottom figures (a) and (b) in Fig. 5 show the displacement plot at $t=22.2 \mu \mathrm{s}$. The absolute value of the displacement vector $|\boldsymbol{u}|=\left|\int \boldsymbol{v} \mathrm{d} t\right|$ is visualized. Although the two figures show the wave field at the same time, the color scale is different. Since the characteristic acoustic impedance $\left(\rho c_{\mathrm{P}}^{[\mathrm{s}]}=1.72 \times 10^{7}\left[\mathrm{~kg} / \mathrm{m}^{2} \mathrm{~s}\right]\right)$ of the solid is much larger than that $\left(\rho c_{\mathrm{P}}^{[\mathrm{a}]}=4.08 \times 10^{2}\left[\mathrm{~kg} / \mathrm{m}^{2} \mathrm{~s}\right]\right)$ of air, it is difficult to see the transmitted wave in aluminum with the same scale as the reflected wave (Fig. 5(a)). When we reduce the value corresponding to the red color, the transmitted wave is visible (Fig. 5(b)). On the other hand, the reflected wave is saturated with the red color at that time. 


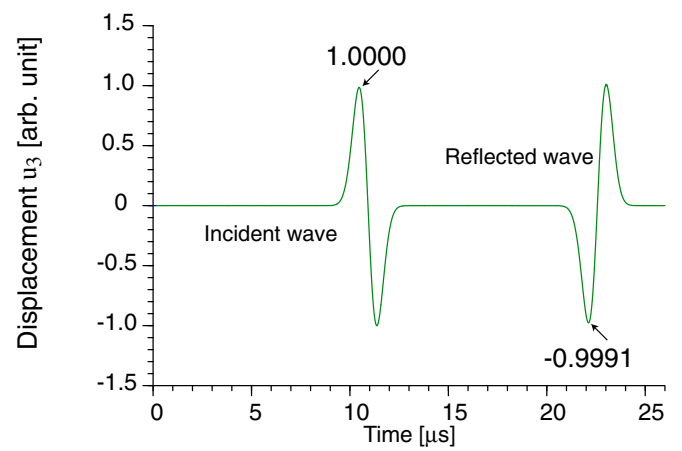

(a) Position A

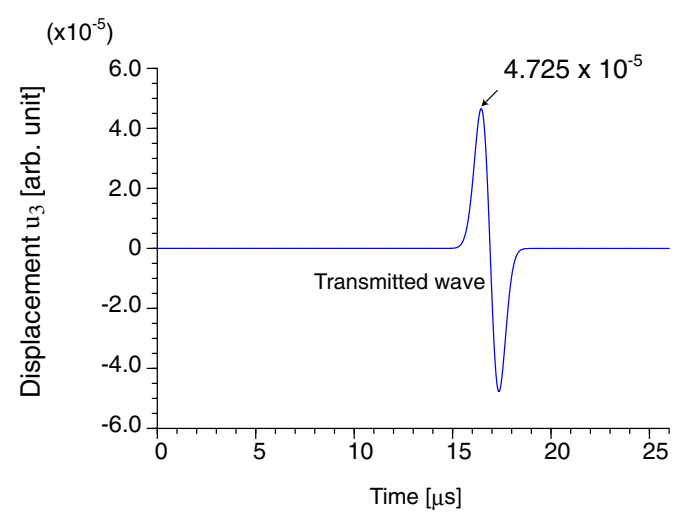

(b) Position B

Fig. 6 (a) Displacement $u_{3}$ calculated at position $\mathrm{A}$ showing the components of the incident and reflected waves. (b) Displacement $u_{3}$ calculated at position B showing the components of the transmitted waves. The displacement in both figures is normalized by the amplitude of the incident wave.

To see the time signals in detail, we output the displacement $u_{3}$ at two positions: $\mathrm{A}$ in the air and $\mathrm{B}$ in the solid. The waveforms at A and B are shown in Figs. 6(a) and $6(\mathrm{~b})$, respectively. The simulation is evaluated by comparing its results with analytical solutions $[17,18]$ for the reflection and transmission coefficients based on the displacement formulation. The reflection coefficient calculated by the FIT simulation is $|-0.9991 / 1.0000|=0.9991$ and the corresponding analytical solution $R$ is 0.99995 as shown in Fig. 7. The transmission coefficient calculated by the FIT simulation is $\left|4.725 \times 10^{-5} / 1.0000\right|=4.725 \times$ $10^{-5}$ and the analytical solution $T_{\mathrm{P}}$ is $4.7518 \times 10^{-5}$. Although the agreement between the analytical solution and the simulation is not perfect, the FIT can model the wave reflection and transmission at the interface with reasonable accuracy.

\section{MODELING OF NON-CONTACT UT}

\subsection{Experimental Setup for Guided Wave Testing}

The experimental setup for the generation and reception of guided waves using air-coupled transducers is shown in Fig. 8. The apparatus consists of two transducers

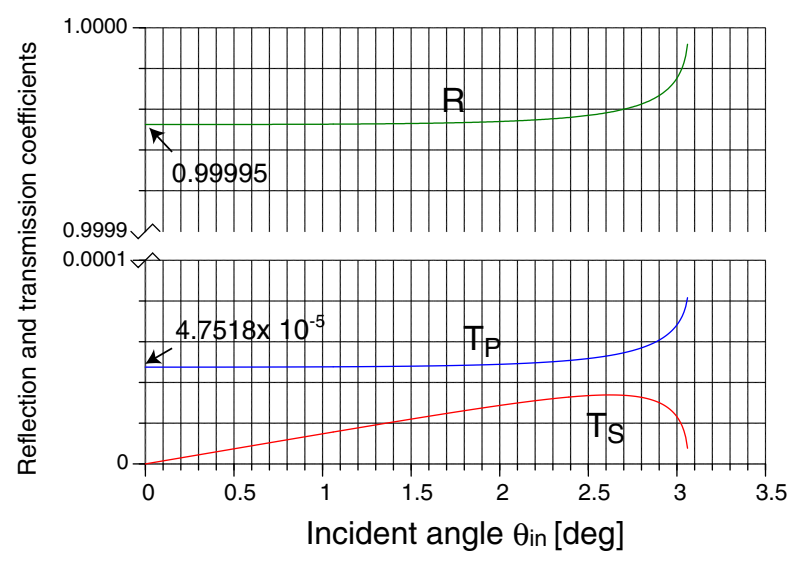

Fig. 7 Analytical solutions of the ultrasonic reflection and transmission for oblique incidence with angle $\theta_{\text {in }}$ onto an air-aluminum interface. The analytical solution is based on the displacement formulation [17]. $R$ represents the reflection coefficient of the P-wave in air. $T_{\mathrm{P}}$ and $T_{\mathrm{S}}$ denote the transmission coefficients of the P-wave in air to the P-wave in the solid and the $\mathrm{P}$-wave in air to the $\mathrm{S}$-wave in the solid, respectively.
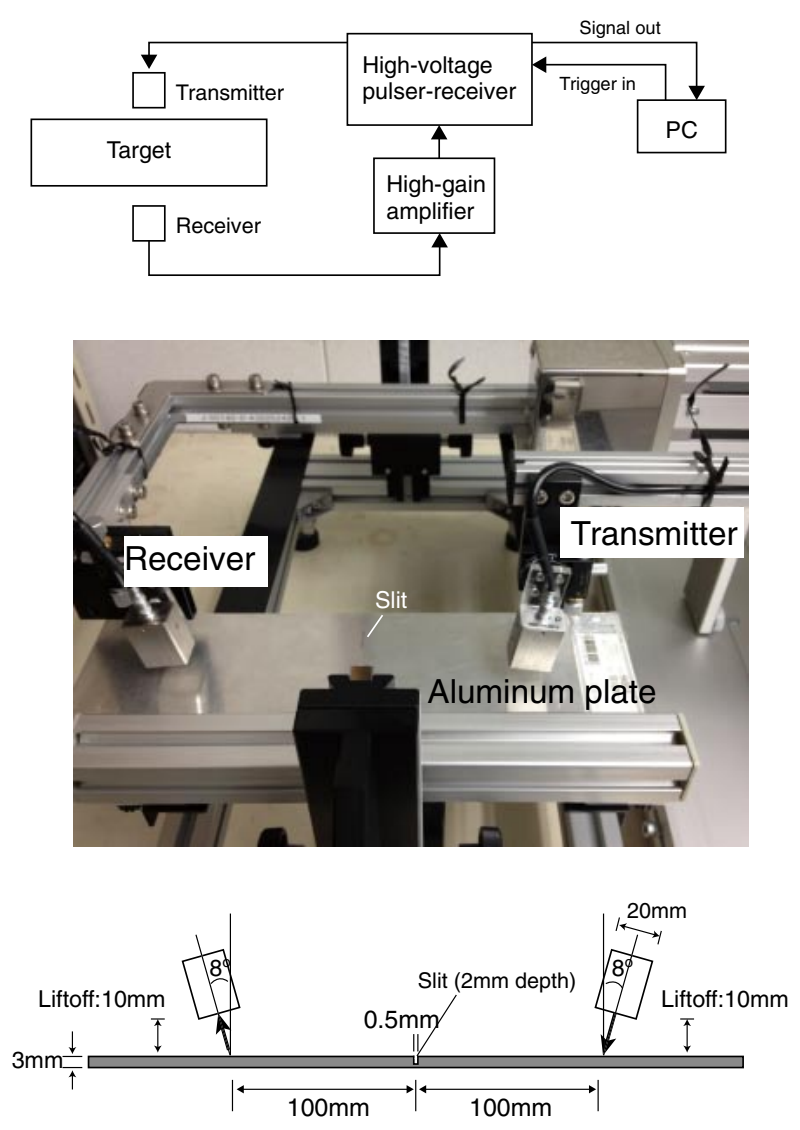

Fig. 8 Setup for guided wave testing with air-coupled transducers. The thickness of the aluminum specimen is $3 \mathrm{~mm}$, and the depth and width of the slit (defect) are 2.0 and $0.5 \mathrm{~mm}$, respectively. 


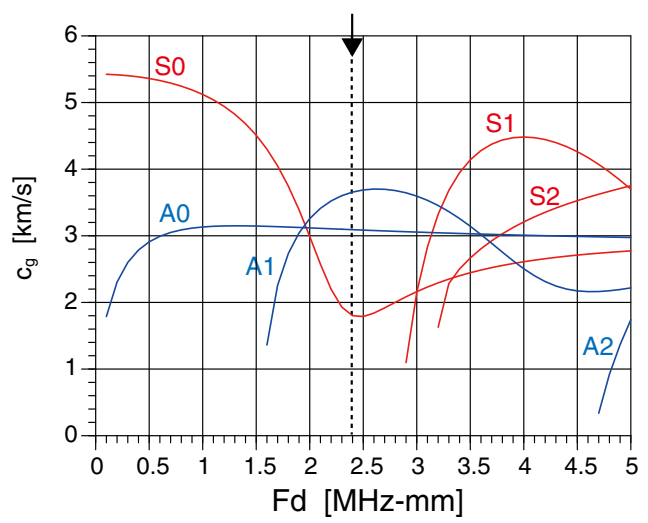

(a)

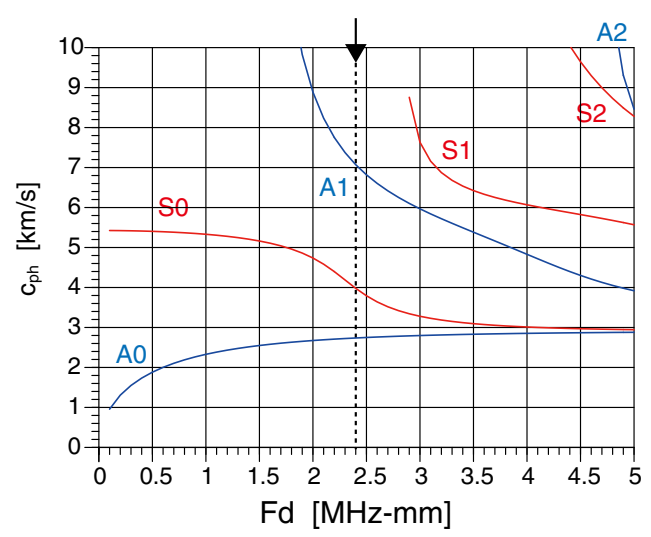

(b)

Fig. 9 Dispersion curves of the group velocity $c_{\mathrm{g}}$ (a) and phase velocity $c_{\mathrm{ph}}(\mathrm{b})$.

(0.8K14X20N, Japan Probe) located in air with a $10 \mathrm{~mm}$ liftoff, a high-voltage pulser/receiver (JPR-10CN, Japan Probe), a gain amplifier (PR-60A, Japan Probe), and a PC to control the signal generation and transducer position. The thickness of the aluminum plate is $3 \mathrm{~mm}$, and the depth and width of the slit (defect) are 2.0 and $0.5 \mathrm{~mm}$, respectively. The aperture of the transducer is $20 \mathrm{~mm}$, and the transducer is excited with an eight-cycle square-wave source with $800 \mathrm{kHz}$ frequency. Dispersion curves of the group velocity $c_{\mathrm{g}}$ and phase velocity $c_{\mathrm{ph}}$ for the aluminum plate are shown in Figs. 9(a) and 9(b), respectively. The curve calculation is based on the equations in [19]. A numerical method to obtain the curves was also proposed by Hayashi and Rose [20]. The horizontal axis is the product of the plate thickness $d$ and frequency $F$. In this measurement, the product $F d$ is approximately $2.4 \mathrm{MHz}-\mathrm{mm}$. Therefore, we find that the three wave components of the symmetric mode (S0) and the antisymmetric modes (A0 and A1 modes) can exist in the plate. The angle of incidence $\theta_{\text {in }}$ in air is obtained from the phase velocity curve and Snell's law:

$$
\sin \theta_{\mathrm{in}}=\frac{c_{\mathrm{in}}}{c_{\mathrm{ph}}} \sin \theta_{\mathrm{ph}}
$$

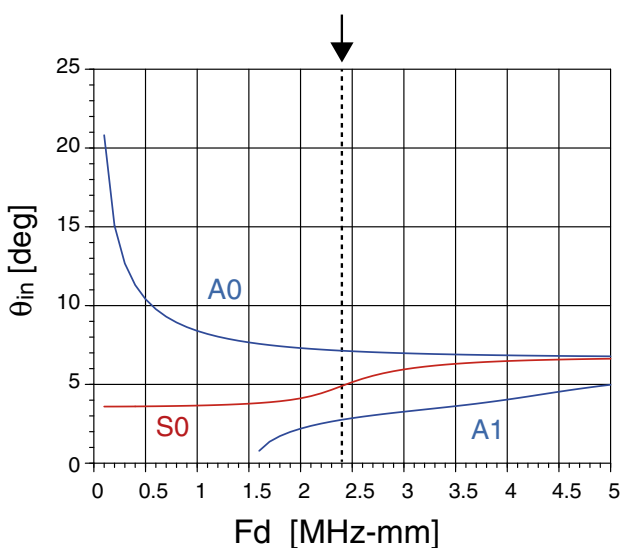

Fig. 10 Incident angle $\theta_{\text {in }}$ calculated using Snell's law and the phase velocity curve.

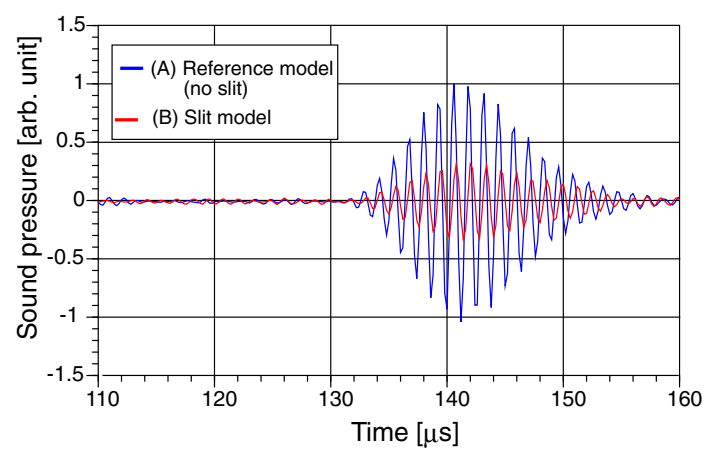

Fig. 11 Waveforms of the A0 mode measured at a receiving air-coupled transducer. The blue and red lines show the waveforms measured in the reference model (no slit) and slit model, respectively.

where $c_{\text {in }}(=340 \mathrm{~m} / \mathrm{s})$ is the wave velocity in air and $\theta_{\mathrm{ph}}$ $\left(=90^{\circ}\right)$ is the angle of propagation of the guided wave in the solid. The incident angles for the S0, A0, and A1 modes, which are calculated using Eq. (15) and the phase velocity curve in Fig. 9(a), are shown in Fig. 10. We find that $8.0^{\circ}$ is an effective angle for the dominant generation of the A0 mode in a solid.

The signals received at the air-coupled transducer are shown in Fig. 11. The blue and red lines show the signals measured in the reference model (no slit) and slit model, respectively. Since the A0 mode propagates at $3100 \mathrm{~m} / \mathrm{s}$ in the plate, both waves can be determined as the A0 mode from the viewpoint of the traveling time. Although the transducer is excited with an eight-cycle square wave, the wave number of the measured signal is more than the number of cycles of excitation. This is due to the electrical circuits in the pulser/receiver and the transformation properties of the electrical signal to the acoustic wave in the transducers, and other reasons. The peak amplitude of the signal in the slit model decreases to $32 \%$ 


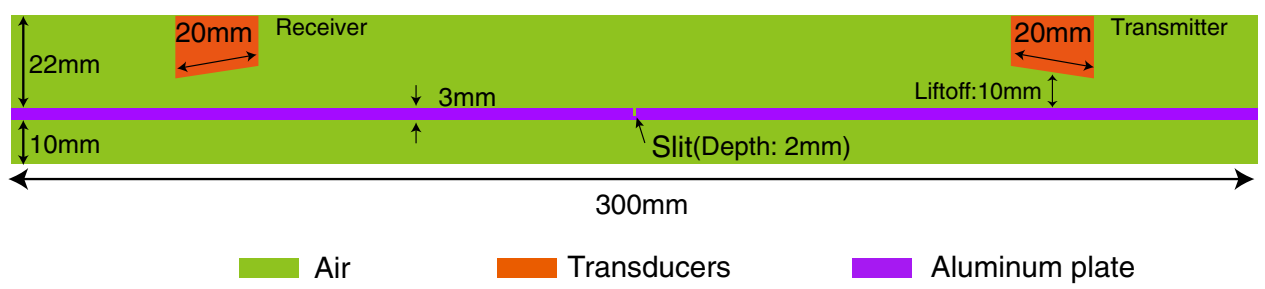

Fig. 12 Calculation model for noncontact UT simulation. The image of this model is constructed using image-based modeling software [21].

of that in the reference model due to the scattering caused by the slit.

\subsection{Simulation of Guided Wave Testing with the Image-based FIT}

Although the noncontact UT experiment in Fig. 8 is, in reality, carried out in $3 \mathrm{D}$ space, the simulation here is solved in the 2D plane strain state in order to decrease the computational load.

We here construct numerical models directly from vector graphics using commercial software [21]. First we draw a digital image, as shown in Fig. 12, which models the measurement setup in Fig. 8. The pixel image is fed into the FIT simulation. The pixel size is $\Delta x=0.01 \mathrm{~mm}$ and the simulation area is a $300 \mathrm{~mm} \times 35 \mathrm{~mm}$ rectangle (total number of pixels is 105 million). The material parameters are $c_{\mathrm{P}}^{[\mathrm{s}]}=6,360 \mathrm{~m} / \mathrm{s}, c_{\mathrm{S}}^{[\mathrm{s}]}=3,040 \mathrm{~m} / \mathrm{s}$, and $\rho=$ $2,700 \mathrm{~kg} / \mathrm{m}^{3}$ for the aluminum, and $c_{\mathrm{P}}^{[\mathrm{a}]}=340 \mathrm{~m} / \mathrm{s}$ and $\rho=1.2 \mathrm{~kg} / \mathrm{m}^{3}$ for air. In this simulation, the model is surrounded by a PML [22]. The geometrical and scattering attenuations are modeled automatically in the FIT simulation. The intrinsic attenuation in both air and aluminum is not considered in this simulation. Here we choose $\Delta t=1.1 \mathrm{~ns}$ by considering the CFL condition. The total number of time steps is 160,000 , i.e., the calculation continues up to $176 \mu \mathrm{s}$. Because it is not easy for us to know the actual shape of transmitted waveforms in air, a modulated tone-burst wave with an $800 \mathrm{kHz}$ frequency is used in this simulation. The incident pressure is distributed uniformly on the transducer surface, and the acoustic wave is transmitted into air.

The visualization of the ultrasonic wave propagation in air and in the aluminum plate is shown in Figs. 13(a)13(d). The color shows the absolute value of the displacement vector $|\boldsymbol{u}|$. The amplitude of the wave transmitted into the aluminum plate is very small due to the large difference between the characteristic acoustic impedances of air and aluminum. Therefore, the value corresponding to the red color in Figs. 13(b)-13(d) is different from that in Fig. 13(a). An acoustic wave is generated at the righthand side of the air-coupled transducer, as shown in Fig. 13(a), and is then transmitted into the aluminum plate
(Fig. 13(b)). The elastic wave of the A0 mode and the leaky waves from the aluminum plate to the air can be observed in Fig. 13(d).

Figure 14 shows the calculated displacement in the reference model. Since a symmetric-mode wave in a plate propagates while maintaining its compressional/tensile motion in the vertical direction, the S0 mode is displayed as a checkerboard pattern in Fig. 14. On the other hand, the A0 mode appears as vertical stripes in Fig. 14 because of the flexural motion. Figure 15 shows the calculated displacement in the model with the slit defect. In Fig. 15, the guided wave of the A0 mode is scattered by the slit, and part of the wave propagates to the left-hand side. The blanks in the leaky wave in the air are due to the multiple scattering between the slit and plate boundaries. The calculated pressure on the surface of the receiving transducer, which is located on the left-hand side of Fig. 12, is plotted in Fig. 16. The blue and red lines show the receiving signals in the reference model and slit model, respectively. From Figs. 11 and 16, the arrival time of the calculated signal is in good agreement with the measured signals. Moreover, the decrease in the peak amplitude in the slit compared with the reference model is about $30 \%$ in the simulation. On the other hand, the measured decrease is $32 \%$. These results show that the image-based FIT possesses acceptable computational accuracy for the simulation of noncontact UT. However, the shapes of the calculated waveforms do not exactly coincide with the measured shapes. In this simulation, we do not characterize the transducer's property regarding the energy transfer from the electrical to the mechanical part, and vice versa. The introduction of more realistic properties in the two transducers will lead to more accurate simulation.

The calculation time was $7.8 \mathrm{~h}$ using three GPUs (Tesla C2075, NVIDIA) mounted in a Linux PC. MPICH 1.4.1p1 was used for the data communication between the GPUs. On the other hand, the calculation time for running the same simulation of a non-GPU code was $96 \mathrm{~h}$ with a multiple core CPU (Xeon E5645, Intel).

\section{CONCLUSIONS}

In order to gain a better understanding of the wave field 

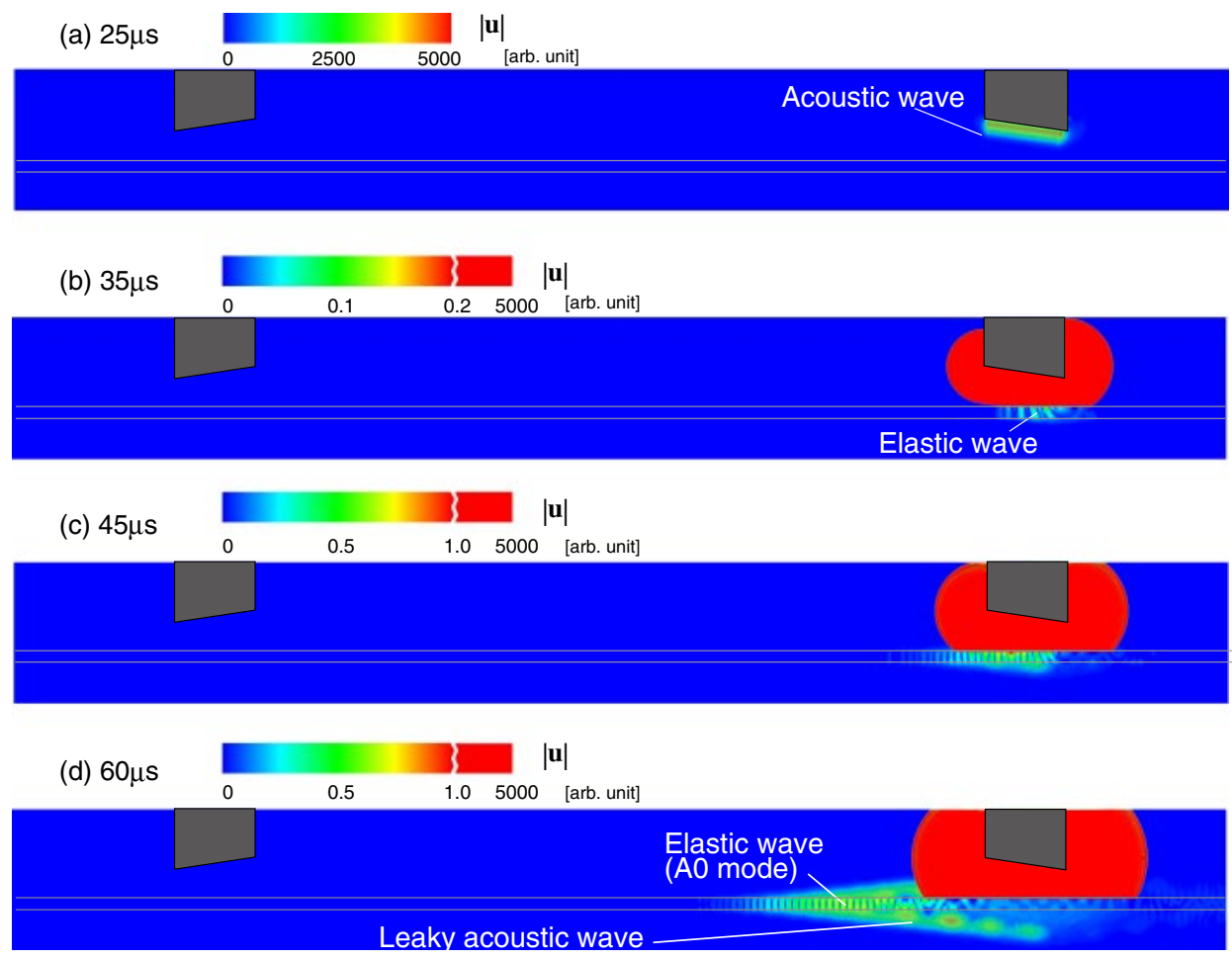

Fig. 13 Calculated displacement amplitude distributions obtained with the image-based FIT. Color images show the absolute value of the displacement vector. An acoustic wave is emitted from an air-coupled transducer at the left-hand side (a), then transmitted into the aluminum plate (b). An elastic wave is generated (c) and leaky acoustic waves are observed (d).

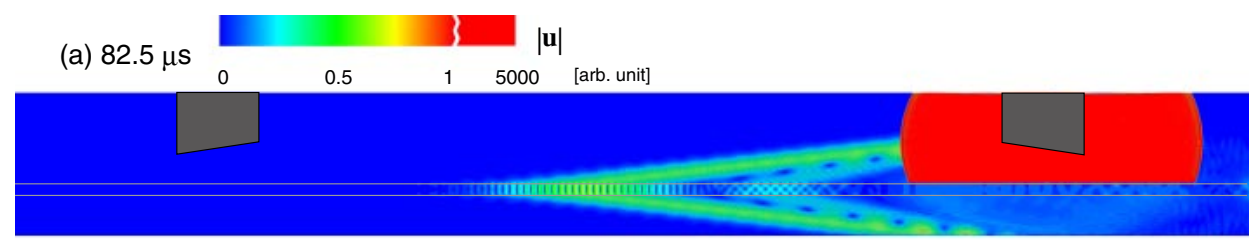

(b) $105 \mu \mathrm{s}$
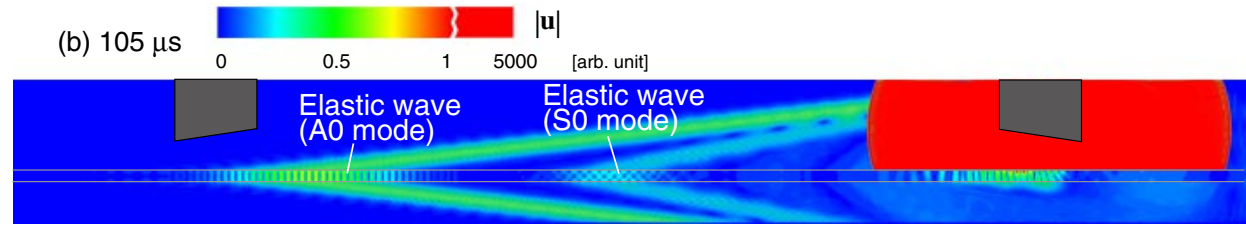

Fig. 14 Calculated displacement amplitude distributions in the reference model. The S0 and A0 modes are displayed as checkerboard patterns and vertical stripes in the plate, respectively.
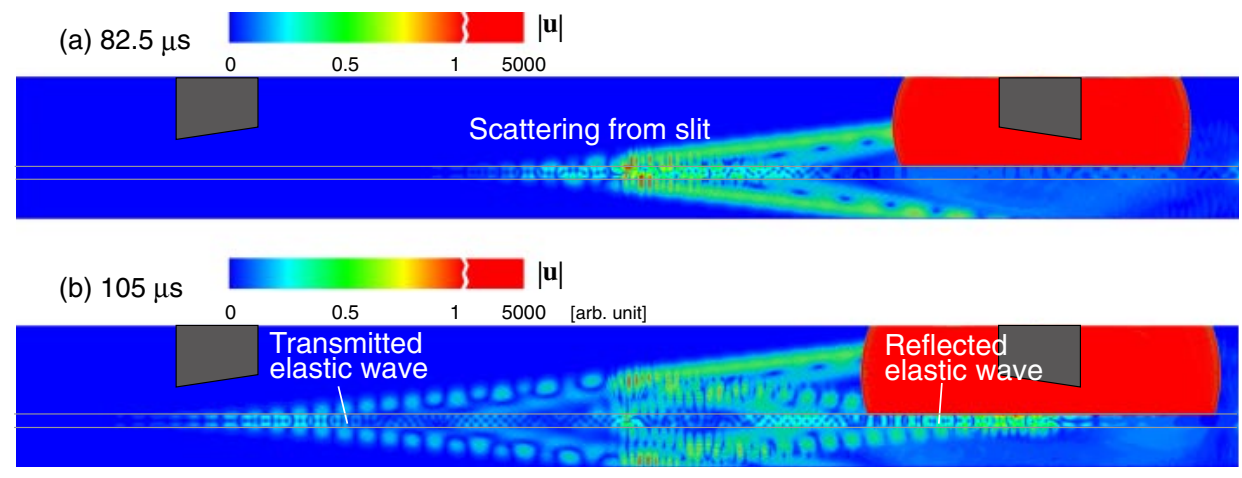

Fig. 15 Calculated displacement amplitude distributions in the slit model. A wave scattered by the slit propagates to the left-hand side. 


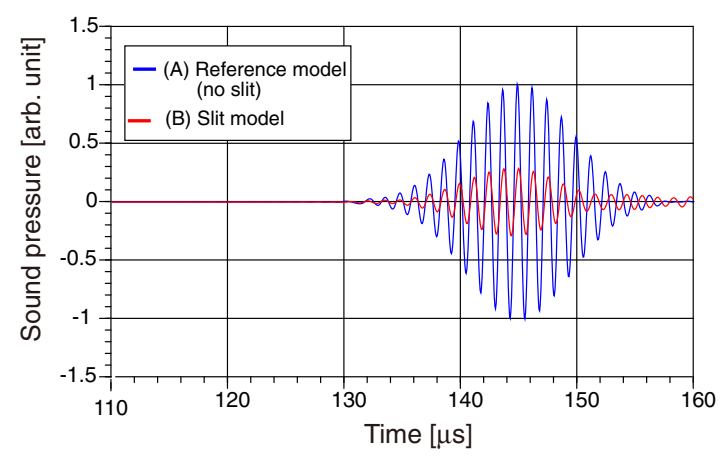

Fig. 16 Waveforms of the A0 mode calculated at the receiving air-coupled transducer. The blue and red lines show the waveforms in the reference model and slit model, respectively.

in noncontact UT, we developed a time domain simulation tool to model wave propagation in air and solids. The tool is based on the FIT and image-based modeling, and the calculation of acoustic and elastic waves can be treated in a unified grid form. Using the image-based modeling, a numerical model can be made directly from a vector graphic and then fed into a FIT simulation.

We first investigated the accuracy of the interface condition in coupled FIT modeling by comparing numerical results with analytical solutions of transmission and reflection coefficients. Our approach was then validated using measured signals in guided wave testing. The results show that the calculated amplitude and arrival time are in good agreement with the measured signals. When simulating this kind of noncontact UT, a fine pixel mesh is required because the wavelength in air is much smaller than that in a solid. To perform the calculation at high speed, we introduce a parallel calculation technique using GPUs. Noncontact UT simulation can be executed within a reasonable time by computing using multiple GPUs.

As future works, we will apply the image-based FIT simulation to various NDT targets and investigate its validity.

\section{REFERENCES}

[1] W. A. Grandia and C. M. Fortunko, "NDE applications of aircoupled ultrasonic transducers," Proc. 1995 IEEE Ultrason. Symp., pp. 697-709 (1995).

[2] M. Castaings and P. Cawley, "The generation, propagation, and detection of Lamb waves in plates using air-coupled ultrasonic transducers," J. Acoust. Soc. Am., 100, 3070-3077 (1996).

[3] S. D. Holland and D. E. Chimenti, "High contrast air-coupled acoustic imaging with zero-group-velocity Lamb modes," Ultrasonics, 42, 957-960 (2004).

[4] C. J. Lissenden and J. L. Rose, "Structural health monitoring of composite laminates through ultrasonic guided wave beam forming," Proc. RTO Applied Vehicle Technology Panel Symp. Military Platform Ensured Availability, RTO-MP-AVT-157
(2008).

[5] H. Nishino, K. Yoshida, T. Asano, Y. Taniguchi, M. Takahashi and Y. Ogura, "Efficient transduction of circumferential Lamb wave by a pair of line focus type noncontact air-coupled ultrasonic transducers and its application for accurate measurement of pipe wall thickness," E-Journal Adv. Mainten., 2, 181190 (2010).

[6] G. Dobie, A. Spencer, K. Burnham, S. G. Pierce, K. Worden, W. Galbraith and G. Hayward, "Simulation of ultrasonic Lamb wave generation, propagation and detection for a reconfigurable air coupled scanner," Ultrasonics, 51, 258-269 (2011).

[7] B. Hosten and C. Biateau, "FE simulation of the generation of guided waves by air-coupled transducers," Rev. Prog. Quantitative Nondestructive Evaluation, AIP Conf. Proc., 975, pp. 47-54 (2008).

[8] K. Nakahata, K. Terada, T. Kyoya, M. Tsukino and K. Ishii, "Simulation of ultrasonic and electromagnetic wave propagation for nondestructive testing of concrete using image-based FIT,” J. Comput. Sci. Technol., 6, 28-37 (2012).

[9] P. Fellinger, R. Marklein, K. J. Langenberg and S. Klaholz, "Numerical modeling of elastic wave propagation and scattering with EFIT - elastodynamic finite integration technique," Wave Motion, 21, 47-66 (1995).

[10] F. Schubert, "Numerical time-domain modeling of linear and nonlinear ultrasonic wave propagation using finite integration techniques - Theory and applications," Ultrasonics, 42, 221229 (2004)

[11] K. Terada, T. Miura and N. Kikuchi, "Digital image-based modeling applied to the homogenization analysis of composite materials," Comput. Mech., 20, 331-346 (1997).

[12] N. Kawada, K. Okubo, N. Tagawa and T. Tsuchiya, "On decreasing the calculation time in multi-dimensional acoustic numerical simulation by multi-GPU parallel computing," Proc. 20th Int. Congr. Acoust., CD-ROM, p841.pdf (2010).

[13] K. Nakahata and K. Kimoto, "Real-time image-based FIT simulation using GPU computing and its application to nondestructive testing," Proc. 6th European Congr. Computational Methods in Applied Sciences and Engineering (ECCOMAS), pp. 4285-4297 (2012).

[14] P. Courant, K. Friedrichs and H. Lewy, "On the partial difference equations of mathematical physics," IBM J. Res. Dev., 11, 215-234 (1967).

[15] The Portland Group, Inc., PGI CUDA FORTRAN Complier, $<$ http://www.pgroup.com/resources/cudafortran.htm> (accessed 1 Aug. 2013).

[16] M. J. Quinn, Parallel Programming in $C$ with MPI and OpenMP (McGraw-Hill, New York, 2003).

[17] L. W. Schmerr, Fundamentals of Ultrasonic Nondestructive Evaluation (Plenum Press, New York, 1998).

[18] J. L. Rose, Ultrasonic Waves in Solid Media (Cambridge University Press, New York, 1999).

[19] T. Kundu, Ultrasonic and Electromagnetic NDE for Structure and Material Characterization: Engineering and Biomedical Applications (CRC Press, Boca Raton, 2012).

[20] T. Hayashi and J. L. Rose, "Guided wave simulation and visualization by a semi analytical finite element method," Mater. Eval., 61, 75-79 (2003).

[21] Japan Probe Co., Ltd., Ultrasonic wave simulator SWAN21, $<$ http://www.jp-probe.com/catalog_pdf/SWAN21.pdf> (accessed 1 Aug. 2013).

[22] F. Collino and C. Tsogka, "Application of the PML absorbing layer model to the linear elastodynamic problem in anisotropic heterogeneous media," Rapport de Recherche, INRIA, N3471 (1998). 